\title{
Asymptotic Frequency Distributions for Variable Coefficients Rayleigh Beams under Boundary Feedback Control
}

\author{
Jun-min Wang ${ }^{\dagger} \quad$ Quoc-Phong Vu ${ }^{\ddagger}$ Siu-Pang Yung ${ }^{\dagger}$ \\ 'Department of Mathematics, The University of Hong Kong, Hong Kong, PRC. \\ wjming@submaths.hku.hk spyung@hku.hk \\ ${ }^{\ddagger}$ Department of Mathematics, Ohio University, Athens, OH45701, U.S.A. \\ qvu@math.ohiou.edu
}

\begin{abstract}
The analysis of the boundary damping rate for eigenmodes of a Rayleigh Beam with variable coefficients is usually difficult because explicit solution formula are hard to come by. In this paper, by using the estimating devices of [9], we can carry out an asymptotic analysis and conclude that there is a uniform damping rate for the high frequency modes. As a result, Riesz basis property and exponential stability can be deduced and a conjecture in [3] is settled.
\end{abstract}

KeyWords: Rayleigh beam, eigenvalue distributions, Riesz basis, strong regularity.

AMS subject classification. $35 \mathrm{P} 20,93 \mathrm{C} 20$

\section{Introduction}

The analysis of boundary damping rate is important in the understanding of vibrating system behavior, such as Riesz basis generation, stabilization, and controllability, etc. In the present paper, we study the following Rayleigh beam model with variable coefficients under the boundary feedback control

$$
\left\{\begin{array}{l}
\rho(x) \frac{\partial^{2} u}{\partial t^{2}}-\frac{\partial}{\partial x}\left(I_{\rho}(x) \frac{\partial^{3} u}{\partial t^{2} \partial x}\right) \\
+\frac{\partial^{2}}{\partial x^{2}}\left(E I(x) \frac{\partial^{2} u}{\partial x^{2}}\right)=0,0<x<1, t>0, \\
u(0, t)=\frac{\partial u}{\partial x}(0, t)=0, t>0, \\
E I \frac{\partial^{2} u}{\partial x^{2}}(1, t)+\alpha \frac{\partial^{2} u}{\partial x \partial t}(1, t)=0, t>0, \\
\frac{\partial}{\partial x}\left(E I \frac{\partial^{2} u}{\partial x^{2}}\right)(1, t)-I_{\rho} \frac{\partial^{3} u}{\partial x \partial t^{2}}(1, t) \\
-\beta \frac{\partial u}{\partial t}(1, t)=0, t>0, \\
u(x, 0)=u_{0}(x), \quad \frac{\partial u}{\partial t}(x, 0)=u_{1}(x) .
\end{array}\right.
$$

Here, $u(x, t)$ is the transverse displacement and $x, t$ stand for the position and time respectively. Also, $\rho(x)>0$ is the mass density, $E I(x)>0$ is the stiffness of the beam, $I_{\rho}(x)>0$ is the mass moment of inertia and $\alpha, \beta \geq 0$ are constant feedback gains that can be tuned. Further details can be found in [1]. Throughout this paper, we always assume that

$$
\rho(x), I_{\rho}(x), E I(x) \in C^{4}[0,1],
$$

and we organize the rest of the paper as follows. In $\S 2$ we use a space-scaling transformation to derive an equivalent eigenvalue boundary problem which is more convenient to expand asymptotically. In $\S 3$ an asymptotic frequency distribution is obtained via expanding the characteristic determinant. In the last section, some important applications of the asymptotic frequency distribution are indicated and a conjecture in [3] is discussed and settled.

\section{Eigenvalue Problem Setup}

Applying the method of separation of variables via $u(x, t):=e^{\lambda t} \phi(x)$, the characteristic equation of the control system (1.1) is given by

$$
\left\{\begin{array}{l}
\lambda^{2} \rho(x) \phi(x)-\lambda^{2}\left(I_{\rho}(x) \phi^{\prime}(x)\right)^{\prime} \\
\quad+\left(E I(x) \phi^{\prime \prime}(x)\right)^{\prime \prime}=0,0<x<1, \\
\phi(0)=\phi^{\prime}(0)=0 \\
E I(1) \phi^{\prime \prime}(1)+\alpha \lambda \phi^{\prime}(1)=0 \\
\left(E I \phi^{\prime \prime}\right)^{\prime}(1)-\lambda^{2} I_{\rho}(1) \phi^{\prime}(1)-\beta \lambda \phi(1)=0
\end{array}\right.
$$

where the sign "w" denotes the derivative with respect to $x$.

We now record down two useful facts for later use.

Lemma 2.1 Let $h_{1}(x), h_{2}(x)$ be two linearly independent solutions for the second order linear homogeneous 
differential equation

$$
\left(I_{\rho}(x) \phi^{\prime}(x)\right)^{\prime}-\rho(x) \phi(x)=0
$$

then we have

$$
D:=h_{1}(0) h_{2}^{\prime}(1)-h_{1}^{\prime}(1) h_{2}(0) \neq 0 .
$$

PROOF. Assume not, then the following system of linear equations

$$
\left\{\begin{array}{l}
t_{1} h_{1}(0)+t_{2} h_{2}(0)=0 \\
t_{1} h_{1}^{\prime}(1)+t_{2} h_{2}^{\prime}(1)=0
\end{array}\right.
$$

is singular because the determinant of the coefficient matrix is $h_{1}(0) h_{2}^{\prime}(1)-h_{1}^{\prime}(1) h_{2}(0)=0$. So there exists a non-trivial solution, say $\left(\begin{array}{l}c_{1} \\ c_{2}\end{array}\right)$. Let $z:=c_{1} h_{1}+c_{2} h_{2}$, then $z$ is a solution of the differential equation with initial conditions:

$$
\left\{\begin{array}{l}
\left(I_{\rho}(x) z^{\prime}(x)\right)^{\prime}-\rho(x) z(x)=0 \\
z(0)=z^{\prime}(1)=0
\end{array}\right.
$$

By the uniqueness theorem of ordinary differential equations, $z \equiv 0$. Thus $h_{1}$ and $h_{2}$ are linearly dependent and this contradicts to the assumption of the lemma.

Lemma 2.2 If $\alpha+\beta>0$, then

$$
\operatorname{Re}(\lambda)<0 \text {. }
$$

PROOF. We go back to the characteristic equation (2.1) of the control system (1.1). Multiplying $\bar{\phi}$, the conjugate of $\phi$, on both side of the first equation in (2.1) and integrating from 0 to 1 with respect to $x$, we obtain

$$
\begin{aligned}
& \lambda^{2} \int_{0}^{1} \rho(x)|\phi(x)|^{2} d x+\lambda^{2} \int_{0}^{1} I_{\rho}(x)\left|\phi^{\prime}(x)\right|^{2} d x \\
& \quad+\beta \lambda|\phi(1)|^{2}+\alpha \lambda\left|\phi^{\prime}(1)\right|^{2} \\
& \quad+\int_{0}^{1} E I(x)\left|\phi^{\prime \prime}(x)\right|^{2} d x=0
\end{aligned}
$$

Let $\lambda=\operatorname{Re} \lambda+i \operatorname{Im} \lambda$, where $\operatorname{Re} \lambda, \operatorname{Im} \lambda$ are real. Then,

$$
\begin{aligned}
& \left((\operatorname{Re} \lambda)^{2}-(\operatorname{Im} \lambda)^{2}\right) \int_{0}^{1}\left(\rho(x)|\phi(x)|^{2}\right. \\
& \left.\quad+I_{\rho}(x)\left|\phi^{\prime}(x)\right|^{2}\right) d x+\beta(\operatorname{Re} \lambda)|\phi(1)|^{2} \\
& +\alpha(\operatorname{Re} \lambda)\left|\phi^{\prime}(1)\right|^{2}+\int_{0}^{1} E I(x)\left|\phi^{\prime \prime}(x)\right|^{2} d x=0
\end{aligned}
$$

and

$$
\begin{aligned}
& 2(\operatorname{Re} \lambda)(\operatorname{Im} \lambda) \int_{0}^{1}\left(\rho(x)|\phi(x)|^{2}+I_{\rho}(x)\left|\phi^{\prime}(x)\right|^{2}\right) d x \\
& +\beta(\operatorname{Im} \lambda)|\phi(1)|^{2}+\alpha(\operatorname{Im} \lambda)\left|\phi^{\prime}(1)\right|^{2}=0 .
\end{aligned}
$$

If $\operatorname{Im} \lambda=0$, then $\operatorname{Re} \lambda<0$ by (2.6). If $\operatorname{Im} \lambda \neq 0$, then $\operatorname{Re} \lambda<0$ follows from (2.7) and the proof is completed.

To simplify further, we expand (2.1) to yield:

$$
\left\{\begin{array}{c}
\phi^{(4)}(x)+2 \frac{E I^{\prime}(x)}{E I(x)} \phi^{\prime \prime \prime}(x)+\frac{E I^{\prime \prime}(x)}{E I(x)} \phi^{\prime \prime}(x) \\
-\lambda^{2}\left(\frac{I_{\rho}(x)}{E I(x)} \phi^{\prime \prime}(x)+\frac{I_{\rho}^{\prime}(x)}{E I(x)} \phi^{\prime}(x)\right. \\
\left.-\frac{\rho(x)}{E I(x)} \phi(x)\right)=0 \\
\phi(0)=\phi^{\prime}(0)=0 \\
E I(1) \phi^{\prime \prime}(1)+\alpha \lambda \phi^{\prime}(1)=0 \\
E I(1) \phi^{\prime \prime \prime}(1)+E I^{\prime}(1) \phi^{\prime \prime}(1) \\
-\lambda^{2} I_{\rho}(1) \phi^{\prime}(1)-\beta \lambda \phi(1)=0
\end{array}\right.
$$

If we introduce a space-scaling transformation (cf. [4, $5,11]$ )

$$
\phi(x):=f(z), \quad z:=\frac{1}{h} \int_{0}^{x}\left(\frac{I_{\rho}(\zeta)}{E I(\zeta)}\right)^{1 / 2} d \zeta
$$

where

$$
h:=\int_{0}^{1}\left(\frac{I_{\rho}(\zeta)}{E I(\zeta)}\right)^{1 / 2} d \zeta
$$

then equation (2.8) can be rewritten as

$$
\left\{\begin{array}{c}
f^{(4)}(z)+a(z) f^{\prime \prime \prime}(z)+b(z) f^{\prime \prime}(z) \\
+c(z) f^{\prime}(z)-h^{2} \lambda^{2}\left[f^{\prime \prime}(z)\right. \\
\left.+d(z) f^{\prime}(z)-e(z) f(z)\right]=0 \\
f(0)=f^{\prime}(0)=0 \\
b_{21} f^{\prime \prime}(1)+b_{22} f^{\prime}(1)+b_{23} \alpha \lambda f^{\prime}(1)=0 \\
b_{11} f^{\prime \prime \prime}(1)+b_{12} f^{\prime \prime}(1)+b_{13} f^{\prime}(1) \\
-\lambda^{2} b_{14} f^{\prime}(1)-\beta \lambda f(1)=0
\end{array}\right.
$$

Here, the sign "'" denotes the derivative in $z$ and

$$
\begin{aligned}
& a(z):= 6 \frac{z_{x x}}{z_{x}^{2}}+2 \frac{1}{z_{x}} \frac{E I^{\prime}(x)}{E I(x)}, \\
& z_{x}= \frac{1}{h}\left(\frac{I_{\rho}(x)}{E I(x)}\right)^{1 / 2}, \\
& b(z):=3 \frac{z_{x x}^{2}}{z_{x}^{4}}+4 \frac{z_{x x x}}{z_{x}^{3}}+6 \frac{z_{x x}}{z_{x}^{3}} \frac{E I^{\prime}(x)}{E I(x)} \\
&+\frac{1}{z_{x}^{2}} \frac{E I^{\prime \prime}(x)}{E I(x)}, \\
& c(z):= \frac{z_{x x x x}}{z_{x}^{4}}+2 \frac{z_{x x x}}{z_{x}^{4}} \frac{E I^{\prime}(x)}{E I(x)} \\
&+\frac{z_{x x}}{z_{x}^{4}} \frac{E I^{\prime \prime}(x)}{E I(x)},
\end{aligned}
$$




$$
\begin{gathered}
d(z):=\frac{z_{x x}}{z_{x}^{2}}+\frac{1}{h^{2} z_{x}^{3}} \frac{I_{\rho}^{\prime}(x)}{E I(x)} \\
e(z):=\frac{1}{h^{2} z_{x}^{4}} \frac{\rho(x)}{E I(x)}
\end{gathered}
$$

and

$$
\begin{aligned}
& b_{11}:=z_{x}^{3}(1) E I(1), \quad b_{14}:=I_{\rho}(1) z_{x}(1), \\
& b_{12}:=3 z_{x}(1) z_{x x}(1) E I(1)+z_{x}^{2}(1) E I^{\prime}(1), \\
& b_{13}:=z_{x x x}(1) E I(1)+z_{x x}(1) E I^{\prime}(1), \\
& b_{21}:=z_{x}^{2}(1) E I(1), \quad b_{22}:=z_{x x}(1) E I(1), \\
& b_{23}:=z_{x}(1) .
\end{aligned}
$$

Now if we replace $\lambda$ by $\mu:=h \lambda$, then (2.11) changes to

$$
\left\{\begin{array}{l}
f^{(4)}(z)+a(z) f^{\prime \prime \prime}(z)+b(z) f^{\prime \prime}(z) \\
+c(z) f^{\prime}(z)-\mu^{2}\left[f^{\prime \prime}(z)\right. \\
\left.\quad+d(z) f^{\prime}(z)-e(z) f(z)\right]=0 \\
f(0)=0 \\
f^{\prime}(0)=0 \\
b_{21} f^{\prime \prime}(1)+b_{22} f^{\prime}(1)+b_{23} \alpha h^{-1} \mu f^{\prime}(1)=0, \\
b_{11} f^{\prime \prime \prime}(1)+b_{12} f^{\prime \prime}(1)+b_{13} f^{\prime}(1) \\
\quad-h^{-2} \mu^{2} b_{14} f^{\prime}(1)-\beta h^{-1} \mu f(1)=0,
\end{array}\right.
$$

which is equivalent to equation $(2.8)$.

\section{Asymptotic Expressions of Eigenfrequencies}

We now divide the complex plane into 4 sectors (for $k=0,1,2,3$ )

$$
\mathcal{S}_{k}:=\left\{z \in \mathbb{C}: \frac{k \pi}{2} \leq \arg z \leq \frac{(k+1) \pi}{2}\right\}
$$

and for each $\mathcal{S}_{k}$, we pick $\omega_{1}$ and $\omega_{2}$ (both square roots of -1) so that

$$
\operatorname{Re}\left(\rho \omega_{1}\right) \leq \operatorname{Re}\left(\rho \omega_{2}\right), \quad \forall \rho \in \mathcal{S}_{k} .
$$

In particular, we can choose

$$
\omega_{1}:=e^{i \frac{\pi}{2}}, \quad \omega_{2}:=e^{i \frac{3}{2} \pi}
$$

in sector $\mathcal{S}_{0}$ and shuffle their orders for other sectors. Writing $\mu=\rho \omega_{1}$ for $\rho$ in each sector $\mathcal{S}_{k}$, we have the following result on the fundamental solutions of (2.19) from [7, Theorem 3] (see also [6]).

Lemma 3.1 For $\rho \in \mathcal{S}_{k}$, with $|\rho|$ sufficiently large, the equation

$$
\begin{array}{r}
f^{(4)}(z)+a(z) f^{\prime \prime \prime}(z)+b(z) f^{\prime \prime}(z)+c(z) f^{\prime}(z) \\
+\rho^{2}\left[f^{\prime \prime}(z)+d(z) f^{\prime}(z)-e(z) f(z)\right]=0
\end{array}
$$

has four linearly independent fundamental solutions $y_{s}(z ; \rho)(s=1,2,3,4)$ and they possess the following asymptotic expressions (for $j=0,1,2,3$ ), if $s=1,2$,

$$
y_{s}^{(j)}(z ; \rho)=h_{s}^{(j)}(z)+\mathcal{O}\left(\rho^{-2}\right)
$$

else $s=3,4$,

$$
y_{s}^{(j)}(z ; \rho)=\left(\rho \omega_{s-2}\right)^{j} e^{\rho \omega_{s-2} x}\left[y_{0}(z)+\mathcal{O}\left(\rho^{-1}\right)\right]
$$

with

$$
y_{0}(z):=e^{-\frac{1}{2} \int_{0}^{z}(a(t)-d(t)) d t} .
$$

Here, $h_{1}(z):=h_{1}(x(z)), h_{2}(z):=h_{2}(x(z))$ are the two linearly independent solutions of (2.2) after the transformation $x(z):=z(x)^{-1}$. That is, they are two linearly independent solutions of

$$
f^{\prime \prime}(z)+d(z) f^{\prime}(z)-e(z) f(z)=0 .
$$

In the sequel, for convenience, we introduce the notation

$$
[a]:=a+\mathcal{O}\left(\rho^{-1}\right) \text {. }
$$

Now the boundary conditions of system (2.19) can be expanded asymptotically by substituting $(3.4),(3.5)$ into them.

Theorem 3.1 Let the boundary conditions of the system (2.19) to be $U_{1}, U_{2}, U_{3}$ and $U_{4}$, then for $\rho \in$ $\mathcal{S}_{0}$, with $|\rho|$ sufficiently large, we have the following asymptotic expansions, .

$$
\begin{aligned}
U_{4}\left(y_{s} ; \rho\right) & =y_{s}(0 ; \rho) \\
& = \begin{cases}h_{s}(0)+\mathcal{O}\left(\rho^{-2}\right), & s=1,2, \\
1+\mathcal{O}\left(\rho^{-1}\right), & s=3,4,\end{cases} \\
& := \begin{cases}{\left[h_{s}(0)\right],} & s=1,2, \\
{[1],} & s=3,4,\end{cases}
\end{aligned}
$$

$$
\begin{aligned}
U_{3}\left(y_{s} ; \rho\right) & =y_{s}^{\prime}(0 ; \rho) \\
& = \begin{cases}x_{z}(0) h_{s}^{\prime}(0)+\mathcal{O}\left(\rho^{-2}\right), & s=1,2 \\
\rho \omega_{s-2}\left(1+\mathcal{O}\left(\rho^{-1}\right)\right), & s=3,4\end{cases} \\
& := \begin{cases}{\left[x_{z}(0) h_{s}^{\prime}(0)\right],} & s=1,2, \\
\rho \omega_{s-2}[1], & s=3,4\end{cases}
\end{aligned}
$$

$$
\begin{aligned}
& U_{2}\left(y_{s} ; \rho\right) \\
= & y_{s}^{\prime \prime}(1 ; \rho)+\frac{b_{22}}{b_{21}} y_{s}^{\prime}(1 ; \rho)+i \frac{b_{23}}{b_{21}} \frac{\alpha}{h} \rho y_{s}^{\prime}(1 ; \rho) \\
= & \left\{\begin{array}{c}
\rho\left(i \frac{\alpha b_{23}}{h b_{21}} x_{z}(1) h_{s}^{\prime}(1)+\mathcal{O}\left(\rho^{-1}\right)\right), s=1,2, \\
\rho^{2} e^{\rho \omega_{s-2}}\left(y_{0}(1) \omega_{s-2}^{2}\right. \\
\left.+i \frac{\alpha b_{23}}{h b_{21}} y_{0}(1) \omega_{s-2}+\mathcal{O}\left(\rho^{-1}\right)\right), s=3,4,
\end{array}\right.
\end{aligned}
$$




$$
\begin{aligned}
:= & \left\{\begin{array}{c}
\rho\left[i \frac{b_{23}}{b_{21}} \frac{\alpha}{h} x_{z}(1) h_{s}^{\prime}(1)\right], \quad s=1,2, \\
\rho^{2} e^{\rho \omega_{s-2}}\left[y_{0}(1) \omega_{s-2}^{2}\right. \\
\left.+i \frac{b_{23}}{b_{21}} \frac{\alpha}{h} y_{0}(1) \omega_{s-2}\right], \quad s=3,4,
\end{array}\right. \\
= & U_{1}\left(y_{s} ; \rho\right) \\
& +\rho^{2} \frac{b_{14}}{h^{2} b_{11}} y_{s}^{\prime}(1 ; \rho)-i \frac{\beta}{h b_{11}} \rho y_{s}(1, \rho) \\
= & \left\{\begin{array}{c}
\rho^{2}\left(\frac{b_{14}}{b_{z}} y_{z}(1)\right. \\
\rho^{3} e^{\rho \omega_{s-2}}\left(y_{0}(1) \omega_{s-2}^{\prime}(1)+\mathcal{O}\left(\rho^{-1}\right)\right), \quad s=1,2 \\
\left.+\frac{b_{14}}{h^{2} b_{11}} y_{0}(1) \omega_{s-2}+\mathcal{O}\left(\rho^{-1}\right)\right), \quad s=3,4,
\end{array}\right. \\
:= & \left\{\begin{array}{c}
\rho^{2}\left[x_{z}(1) h_{s}^{\prime}(1)\right], \\
\rho^{3} e^{\rho \omega_{s-2}}\left[y_{0}(1) \omega_{s-2}^{3}\right. \\
\left.+y_{0}(1) \omega_{s-2}\right],
\end{array} \quad s=1,2,\right.
\end{aligned}
$$

PROOF. It is a direct computation. Note that in (3.10),

$$
\frac{b_{14}}{b_{11}}=\frac{I_{\rho}(1) z_{x}(1)}{z_{x}^{3}(1) E I(1)}=h^{2} .
$$

To estimate the eigenvalues, we substitute the above expansions (3.7), (3.8), (3.9), (3.10) into the characteristic determinant $\Delta(\rho)=$

$$
\left|\begin{array}{llll}
U_{4}\left(y_{1}, \rho\right) & U_{4}\left(y_{2}, \rho\right) & U_{4}\left(y_{3}, \rho\right) & U_{4}\left(y_{4}, \rho\right) \\
U_{3}\left(y_{1}, \rho\right) & U_{3}\left(y_{2}, \rho\right) & U_{3}\left(y_{3}, \rho\right) & U_{3}\left(y_{4}, \rho\right) \\
U_{2}\left(y_{1}, \rho\right) & U_{2}\left(y_{2}, \rho\right) & U_{2}\left(y_{3}, \rho\right) & U_{2}\left(y_{4}, \rho\right) \\
U_{1}\left(y_{1}, \rho\right) & U_{1}\left(y_{2}, \rho\right) & U_{1}\left(y_{3}, \rho\right) & U_{1}\left(y_{4}, \rho\right)
\end{array}\right|
$$

and make use of the fact that the zeros of $\Delta(\rho)$ are the eigenvalues of (2.1) (cf. [9, pp.13-15]).

Theorem 3.2 In sector $\mathcal{S}_{0}$, the characteristic determinant $\Delta(\rho)$ of the characteristic equation (2.19) has an asymptotic expansion

$$
\begin{array}{r}
\Delta(\rho)=-i \rho^{5} y_{0}(1) x_{z}(1) D\left\{e^{-i \rho}(1-\alpha \gamma)\right. \\
\left.+e^{i \rho}(1+\alpha \gamma)+\mathcal{O}\left(\rho^{-1}\right)\right\}
\end{array}
$$

where $\gamma:=\left(I_{\rho}(1) E I(1)\right)^{-1 / 2}, D:=\left(h_{2}^{\prime}(1) h_{1}(0)-\right.$ $\left.h_{1}^{\prime}(1) h_{2}(0)\right)$ the nonizero determinant defined in (2.3). Furthermore, the boundary problem (2.19) is strongly regular in the sense of $[8, p .259]$ iff the following condition holds:

$$
1-\alpha\left(I_{\rho}(1) E I(1)\right)^{-1 / 2} \neq 0 \quad(\text { i.e. } 1-\alpha \gamma \neq 0) .
$$

PROOF. In sector $\mathcal{S}_{0}$, with $\omega_{1}:=i, \omega_{2}:=-i$, we conclude that (for $s=3,4$,)

$$
\begin{gathered}
U_{1}\left(y_{s}, \rho\right)=\rho^{3} e^{\rho \omega_{s-2}}[0], \\
U_{2}\left(y_{s}, \rho\right)=\rho^{2} e^{\rho \omega_{s-2}}\left[-y_{0}(1)+(-1)^{s} \frac{b_{23}}{b_{21}} \alpha h^{-1} y_{0}(1)\right] .
\end{gathered}
$$

Substituting (3.7), (3.8), (3.9), (3.10), (3.14), (3.15) into the characteristic determinant, we have $\Delta(\rho)=$

$$
\begin{aligned}
& \operatorname{det}\left(\begin{array}{c}
{\left[h_{1}(0)\right]} \\
{\left[x_{z}(0) h_{1}^{\prime}(0)\right]} \\
\rho\left[i \frac{b_{23}}{b_{21}} \alpha h^{-1} x_{z}(1) h_{1}^{\prime}(1)\right] \\
\rho^{2}\left[x_{z}(1) h_{1}^{\prime}(\mathbf{1})\right]
\end{array}\right. \\
& {\left[h_{2}(0)\right]} \\
& {\left[x_{z}(0) h_{2}^{\prime}(0)\right]} \\
& \rho\left[i \frac{b_{23}}{b_{21}} \alpha h^{-1} x_{z}(1) h_{2}^{\prime}(1)\right] \\
& \rho^{2}\left[x_{z}(1) h_{2}^{\prime}(1)\right] \\
& \text { [1] } \\
& \text { i } \rho[1] \\
& \rho^{2} e^{\rho \omega_{1}}\left[\begin{array}{c}
\left.-y_{0}(1)-\frac{b_{23}}{b_{21}} \alpha h^{-1} y_{0}(1)\right] \\
\rho^{3} e^{\rho \omega_{1}[0]}
\end{array}\right. \\
& -i p[1] \\
& \rho^{2} e^{\rho \omega_{2}}\left[\begin{array}{c}
-y_{0}(1)+\frac{b_{23}}{b_{21}} \alpha h^{-1} \\
\left.y_{0}(1)\right] \\
\rho^{3} e^{\rho \omega_{2}}[0]
\end{array}\right) \\
& =-i \rho^{5} y_{0}(1) x_{\tau}(1) D\left\{e^{\rho \omega_{2}}\left[1-\frac{b_{23}}{b_{21}} \alpha h^{-1}\right]\right. \\
& \left.+e^{\rho \omega_{1}}\left[1+\frac{b_{23}}{b_{21}} \alpha h^{-1}\right]\right\} \text {. }
\end{aligned}
$$

Combining with $(2.9),(2.18)$, we have

$$
\frac{b_{23}}{b_{21}}=\frac{z_{x}(1)}{z_{x}^{2}(1) E I(1)}=h\left(I_{\rho}(1) E I(1)\right)^{-1 / 2}=h \gamma \text {. }
$$

Thus, the asymptotic expansion (3.12) of $\Delta(\rho)$ is obtained. The strong regularity of $[8$, Def.2.7] can be verified directly from the fact that $y_{0}(1), x_{z}(1)>0$ and $(2.3),(3.13)$.

Remark 3.1 From [9, pp.57-74], we see that expression (3.12) also holds in other sectors $\mathcal{S}_{k}$ under the same argument as in sector $\mathcal{S}_{0}$ and conclude that the set of eigenvalues in sectors $\mathcal{S}_{1}$ and $\mathcal{S}_{3}$ are the same as those in $\mathcal{S}_{0}$ and $\mathcal{S}_{2}$.

Remark 3.2 Since equation (2.1) is equivalent to (2.11), so (2.1) is also strongly regular under condition (3.13) in the sense of $[8$, Def.2.7]. This strong regularity is very important in applications (see Remark 4.1). 
Theorem 3.3 Suppose that the problem (2.1) is strongly regular (that is, the condition (3.13) is fulfilled), then the eigenvalues $\lambda_{k}$ of the problem (2.1) have the following asymptotic behavior

$$
\lambda_{k}=\frac{1}{h}\left(\frac{1}{2} \xi_{0}+k \pi i\right)+\mathcal{O}\left(k^{-1}\right), \quad k= \pm 1, \pm 2, \ldots
$$

where $h:=\int_{0}^{1}\left(\frac{I_{\rho}(\zeta)}{E I(\zeta)}\right)^{1 / 2} d \zeta$ defined in (2.10) and

$$
\xi_{0}:= \begin{cases}\ln \frac{\alpha \gamma-1}{\alpha \gamma+1}, & \alpha \gamma>1 \\ \ln \frac{1-\alpha \gamma}{1+\alpha \gamma}+\pi i, & \alpha \gamma<1\end{cases}
$$

It is also clear to see that

$$
\operatorname{Re} \xi_{0}=\ln \left|\frac{\alpha \gamma-1}{\alpha \gamma+1}\right|<0
$$

and

$$
\operatorname{Re} \lambda_{k} \rightarrow \frac{1}{2 h} \operatorname{Re} \xi_{0}<0, \quad k \rightarrow \infty
$$

PROOF. In sector $\mathcal{S}_{0}$, since the boundary problem (2.1) is strongly regular and $y_{0}(1), x_{z}(1)>0$, by (3.12), (2.3) and $\Delta(\rho)=0$, we have

$$
e^{-i \rho}(1-\alpha \gamma)+e^{i \rho}(1+\alpha \gamma)+\mathcal{O}\left(\rho^{-1}\right)=0 .
$$

If we consider the equation

$$
e^{-i \rho}(1-\alpha \gamma)+e^{i \rho}(1+\alpha \gamma)=0
$$

then, since $1+\alpha \gamma>0$, we get

$$
e^{2 i \rho}=\frac{\alpha \gamma-1}{1+\alpha \gamma}
$$

and come up with solutions

$$
\tilde{\mu}_{k}=i \rho_{k}=\frac{1}{2} \xi_{0}+k \pi i, \quad k=1,2, \ldots
$$

with $\xi_{0}$ defined in (3.18). Apply (3.21) back to (3.20) through Rouche's theorem and we obtain

$$
\mu_{k}=\frac{1}{2} \xi_{0}+k \pi i+\mathcal{O}\left(k^{-1}\right), \quad k=0,1,2, \ldots
$$

In sector $\mathcal{S}_{2}$, we can argue similarly on the asymptotic distribution of the conjugate $\bar{\mu}_{k}$. Here in order to satisfy (3.2), we let $\omega_{1}:=-i, \omega_{2}:=i$ and then equations (3.8), (3.9), (3.10), (3.14) and (3.15) change to (recall that $\left.\mu=\rho \omega_{1}=-i \rho\right)$

$$
U_{3}\left(y_{s} ; \rho\right)= \begin{cases}\left(x_{z}(0) h_{s}^{\prime}(0)\right], & s=1,2 \\ (-1)^{s} i \rho[1], & s=3,4\end{cases}
$$

$$
\begin{aligned}
& U_{2}\left(y_{s} ; \rho\right)=\left\{\begin{array}{cc}
\rho\left[-i \frac{b_{23}}{b_{21}} \frac{\alpha}{h} x_{z}(1) h_{s}^{\prime}(1)\right], & s=1,2, \\
\rho^{2} e^{\rho \omega_{s-2}}\left[-y_{0}(1)\right. & \\
\left.+(-1)^{s} \frac{b_{23}}{b_{21}} \frac{\alpha}{h} y_{0}(1)\right], & s=3,4,
\end{array}\right. \\
& U_{1}\left(y_{s} ; \rho\right)= \begin{cases}\rho^{2}\left[x_{z}(1) h_{s}^{\prime}(1)\right], & s=1,2, \\
\rho^{3} e^{\rho \omega_{s-2}}[0], & s=3,4 .\end{cases}
\end{aligned}
$$

Thus we have,

$$
\begin{aligned}
\Delta(\rho)= & i \rho^{5} y_{0}(1) x_{z}(1) D\left\{e^{i \rho}(1-\alpha \gamma)\right. \\
& \left.+e^{-i \rho}(1+\alpha \gamma)+\mathcal{O}\left(\rho^{-1}\right)\right\}
\end{aligned}
$$

Furthermore $\Delta(\rho)=0$ becomes

$$
e^{i \rho}(1-\alpha \gamma)+e^{-i \rho}(1+\alpha \gamma)+\mathcal{O}\left(\rho^{-1}\right)=0,
$$

and so

$$
\bar{\mu}_{k}=\frac{1}{2} \xi_{0}-k \pi i+\mathcal{O}\left(k^{-1}\right), \quad k=1,2, \ldots
$$

Hence, we can conclude from (3.23) and (3.29) that

$$
\mu_{k}=\frac{1}{2} \xi_{0}+k \pi i+\mathcal{O}\left(k^{-1}\right), \quad k= \pm 1, \pm 2, \ldots
$$

Since $\mu=h \lambda$, so $\lambda_{k}=\frac{1}{h} \mu_{k}=$

$$
\frac{1}{h}\left(\frac{1}{2} \xi_{0}+k \pi i\right)+\mathcal{O}\left(k^{-1}\right), \quad k= \pm 1, \pm 2, \ldots
$$

From Remark 3.1, we see that the set of eigenvalues in $\mathcal{S}_{3}$ and $\mathcal{S}_{4}$ are the same as those in $\mathcal{S}_{0}$ and $\mathcal{S}_{2}$ and so the proof is completed.

\section{Applications}

We now describe some interesting applications of our main results. The details can be found in [10].

Remark 4.1 Assume that the boundary problem (2.1) is strongly regular (that is, the inequality (3.13) is fulfilled), then Theorem 3.3 implies that the system of the eigenfunctions $\left\{\left(\phi_{k}, \lambda_{k} \phi_{k}\right): k \in \mathbb{Z}\right\}$ forms a Riesz basis on the energy Hilbert space

$$
\begin{aligned}
\mathbf{H}:= & \left\{f \in H^{2}(0,1) \mid f(0)=f^{\prime}(0)=0\right\} \\
& \times\left\{f \in H^{1}(0,1) \mid f(0)=0\right\} .
\end{aligned}
$$

Hence, the spectrum-determined growth condition will be true and (3.19).(2.4) will yield asymptotic stability for $\alpha+\beta>0$ and exponential stability for $\alpha>$ $0,1-\alpha \gamma \neq 0$, respectively, and we refer the interested readers to [10] for details. These results are very interesting because they apply to Rayleigh Beams with variable coefficients, not just constant coefficients. 
Remark 4.2 The special case that $\rho(x)=E I(x) \equiv 1$ and $I_{\rho}(x) \equiv \gamma_{1}>0$ was discussed in [2], [3]. In this case, expression (3.17) then becomes (for $k=$ $0, \pm 1, \pm 2, \ldots)$

$$
\lambda_{k}=\frac{1}{\sqrt{\gamma_{1}}}\left(\frac{1}{2} \xi_{1}+k \pi i\right)+\mathcal{O}\left(k^{-1}\right),
$$

with

$$
\xi_{1}= \begin{cases}\ln \frac{\alpha-\sqrt{\gamma_{1}}}{\alpha+\sqrt{\gamma_{1}}}, & \alpha>\sqrt{\gamma_{1}}, \\ \ln \frac{\sqrt{\gamma_{1}}-\alpha}{\alpha+\sqrt{\gamma_{1}}}+\pi i, & \alpha<\sqrt{\gamma_{1}}\end{cases}
$$

and

$$
\operatorname{Re} \lambda_{k} \rightarrow \frac{1}{2 \sqrt{\gamma_{1}}} \ln \left|\frac{\alpha-\sqrt{\gamma_{1}}}{\alpha+\sqrt{\gamma_{1}}}\right|<0, \quad k \rightarrow \infty .
$$

So the closer $\alpha$ to $\alpha^{*}:=\sqrt{\gamma_{1}}$ the larger the damping rate for the system (1.1) which is the conjecture made in [3]. However, when we set the control gain $\alpha=\sqrt{\gamma_{1}}$, then $\sigma(\mathcal{A})$ contains only finitely many eigenvalues. This is because $\mathcal{A}$ has compact resolvents, so there are only finitely many eigenvalues inside each bounded set and (3.12) tells us that there are no eigenvalues at all when their moduli are large enough.

\section{Acknowledgement}

This work is supported by an RGC grant of code HKU $7133 / 02 \mathrm{P}$.

\section{References}

[1] D. L. Russell, Mathematical models for the elastic beam and their control-theoretic implications, Semigroups, theory and applications, Vol. II, Longman Sci. Tech., Harlow, (1986) 177-216.

[2] B. P. Rao, A compact perturbation method for the Boundary stabilization of the Rayleigh beam equation, Applied Mathematics and Optimization, Vol. 33, (1996) 253-264.

[3] B. Z. Guo, Basis property of a Rayleigh beam with boundary stabilization, Journal of Optimization Theory and Applications, Vol. 112, No.3 (2002) 529547.

[4] B. Z. Guo, Riesz basis property and exponential stability of controlled Euler-Bernoulli beam equations with variable coefficients, SIAM J.Control \& Optim., Vol. 40 (2002), 1905-1923.

[5] B. Z. Guo, On the boundary control of a Hybrid system with variable coefficients, Journal of optimization theory and applications, Vol. 114, No.2 (2002) 373395.
[6] W. Eberhard and G. Freiling, Stone-reguläre Eigenwertprobleme, Math.Z., Vol. 160 (1978) 139-161.

[7] C. Tretter, On fundamental systems for differential equations of Kamke type, Math. Z., Vol. 219 (1995) 609-629.

[8] C. Tretter, Kamke problems. Properties of the eigenfunctions, Math. Nachr., Vol. 170 (1994) 251-275.

[9] M. A. Naimark, Linear Differential Operators, Vol.I, Frederick Ungar Publishing Company, New York, 1967.

[10] J. M. Wang, G. Q. Xu and S. P. Yung, Exponential stability for variable coefficients rayleigh beams under boundary feedback control, to appear.

[11] Z. Liu, Boundary Stabilization of Nonhomogeneous Beam by Frequency Domain Multiplier Method, 4th SIAM conf. on control and its applications, May $7-9,1998$. 